\title{
THE ABSOLUTE FORWARD PHOTODISINTEGRATION CROSS SECTION OF THE DEUTERON AT LOW EXCITATION ENERGIES
}

\author{
A. DE GRAEVE ${ }^{\text {a }}$, A. ZIEGER ${ }^{\text {b }}$, R. VAN DE VYVER ${ }^{a}$, H. FERDINANDE ${ }^{a}$, \\ L. VAN HOOREBEKE ${ }^{a}$, D. RYCKBOSCH ${ }^{a}$, P. VAN OTTEN ${ }^{a}$ and B. ZIEGLER ${ }^{b}$ \\ a Nuclear Physics Laboratory, RUG, B-9000 Ghent, Belgium \\ b Abteilung Kernphysik, Max Planck Institut für Chemie, D-6500 Mainz, FRG
}

Received 25 April 1989

\begin{abstract}
The ${ }^{2} \mathrm{H}(\gamma, \mathrm{p}) \mathrm{n}$ cross section was measured absolutely at $0^{\circ}$ for the outgoing proton, for photon energies between 8 and $18 \mathrm{MeV}$. The rising trend observed in the cross section in this energy region indicates the existence of the theoretically predicted minimum. Our results at $E_{y} \approx 10 \mathrm{MeV}$ are well described by recent relativistic calculations, the data around $15 \mathrm{MeV}$, on the contrary, are commonly underestimated.
\end{abstract}

The understanding of the photodisintegration of the deuteron, even at low excitation energies $(<140$ $\mathrm{MeV}$ ) is of fundamental importance for the knowledge of the nucleon-nucleon interaction and of the electromagnetic transition operator, and as a consequence, the deuteron represents one of the most extensively studied nuclei. In spite of this, there still is much interest in this photodisintegration process as up till now no conclusive description has been given for it, neither theoretically nor experimentally.

For some years now, it has been clear that Partovi's [1] nonrelativistic impulse approximation calculation fails to reproduce the deuteron photodisintegration cross section, especially at extreme angles [2]. Although the forward cross section could be lowered by reducing the $D$-state probability in the ground state of the deuteron [3], such a procedure eventually leads to unrealistic values for related quantities; neither can the inclusion of contributions from meson exchange currents (MEC) or from isobaric configurations (IC) to the transition operator [4] remove the discrepancy between theory and experiment over the entire, measured energy range. Only recently the importance of relativistic corrections to the transition operator was recognized; especially the relativistic spin-

\footnotetext{
Supported in part by the Deutsche Forschungsgemeinschaft
} (SFB 201). orbit coupling was found to produce a remarkable improvement in the description of the reaction at extreme angles [5-10]. Some problems concerning the consistency of such relativistic treatments, however, remain to be solved.

From the experimental side, the interest in this specific reaction was triggered by the results from the pioneering work by Hughes et al. [2], who measured the forward differential cross section in the photon energy interval from 20 to $120 \mathrm{MeV}$. These data were independently confirmed by various (mostly radiative capture) measurements [11-16]. Furthermore, a recent measurement [17] of this cross section at $10.74 \mathrm{MeV}$ photon energy aimed at the verification of the existence of the theoretically predicted minimum in that energy region.

The accurate experiment reported on in this letter actually represents a continuation of the research started by Zieger et al. [17]; the measurements were performed at the $90 \mathrm{MeV}$ electron linac of the Gent State University and cover the photon range from 9 to $18 \mathrm{MeV}$.

After passing through a beam hardener, the well collimated bremsstrahlung photon beam impinged on the target, beyond which a magnetic translation system was positioned in order to deflect the produced protons out of the photon beam line. As a target a $(94.2 \%)$ deuterated polyethylene foil was used with 
a total thickness of $9.76 \mathrm{mg} / \mathrm{cm}^{2}$, while the background was measured with a $9.11 \mathrm{mg} / \mathrm{cm}^{2}$ thick pure polyethylene sheet. Beyond this target, a $5 \mathrm{~mm}$ thick Ta collimator $\left(\varnothing_{\text {in }}=20 \mathrm{~mm}\right)$ was located, confining the forward going particles to a cone with a half-angle of $21.8 \mathrm{mrad}$.

Apart from a few minor modifications, the translation system is essentially the same as in ref. [17]. It consists of two dipole magnets placed in a non-dispersive mode, which focus the accepted photoprotons upon a (fully depleted) large area Si surface barrier detector. In between both magnets an additional collimator (with a rectangular aperture of $5 \mathrm{~cm}$ high $\times 12 \mathrm{~cm}$ wide) was inserted, mainly with the purpose of suppressing the in-chamber background.

The detector itself was energy calibrated using a ${ }^{228} \mathrm{Th} \alpha$-source. As the experimental conditions for the measurements performed at 10 and $15 \mathrm{MeV}$ photon energy were not identical, the relevant information is summarized in table 1 . Finally, the photon beam flux was absolutely monitored with an NBS-P2 ionization chamber.

The transmission of the magnet system, which is important for the knowledge of the geometric efficiency of the detection system, was calculated using a full ray-tracing computer code (imbedded in a Monte Carlo procedure) and measured with the help of an absolutely calibrated $\alpha$-source. Fig. 1, displaying the geometric efficiency of the system for the 10 $\mathrm{MeV}$ experiment, shows that the agreement between both results is excellent in the plateau region while some minor deviations can be observed in the (more sensitive) slopes of the curve. The accepted range $\Delta p /$ $p$ equals about $20 \%$.

Concerning the actual data taking, several runs were performed at each magnet setting (see table 1) with the $\mathrm{CD}_{2}$ and the $\mathrm{CH}_{2}$ targets. The result from the

\section{Table 1}

Experimental parameters for the forward ${ }^{2} \mathrm{H}(\gamma, \mathrm{p})$ n measurement at photon energies of about 10 and $15 \mathrm{MeV}$.

\begin{tabular}{|c|c|c|c|c|}
\hline \multirow{2}{*}{$\begin{array}{l}\left\langle E_{\gamma}\right\rangle \\
(\mathrm{MeV})\end{array}$} & \multirow{2}{*}{$\begin{array}{l}T_{-}(\mathrm{MeV}) \\
\text { endpoint }\end{array}$} & \multirow{2}{*}{$\begin{array}{l}p_{0}(\mathrm{MeV} / \mathrm{c}) \\
\text { focusing } \\
\text { momentum }\end{array}$} & \multicolumn{2}{|c|}{ Si detector } \\
\hline & & & $\begin{array}{l}\text { area } \\
\left(\mathrm{mm}^{2}\right)\end{array}$ & $\begin{array}{l}\text { thickness } \\
(\mu \mathrm{m})\end{array}$ \\
\hline 10 & 18 & 85 & 2000 & 300 \\
\hline 15 & 20 & 115 & 1250 & 2000 \\
\hline
\end{tabular}

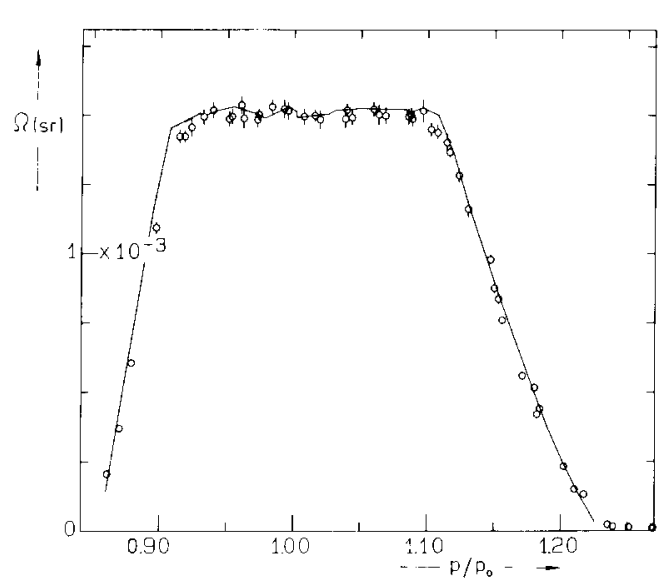

Fig. 1. Comparison of the measured geometric efficiency of the detection system (data points) with the calculated one (full line), as a function of $p / p_{0}$, with $p_{0}$ the focusing momentum.

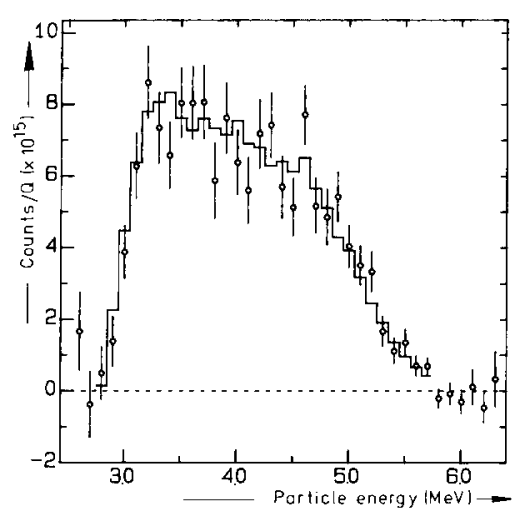

Fig. 2. The net spectrum at $\left\langle E_{\gamma}\right\rangle=10 \mathrm{MeV}$, resulting from a straightforward subtraction of the $\left(\mathrm{CH}_{2}\right)$ background from the foreground spectrum. The histogram represents the calculated energy distribution as described in the text.

measurements was found to be very clean: the low energetic exponential background could always be clearly separated from the true counts originating from the target. The net counts are obtained by subtracting the $\mathrm{CH}_{2}$ background from the $\mathrm{CD}_{2}$ signal, normalized to the same amount of charge collected in the $\mathbf{P} 2$ ionization chamber. An example of such a net spectrum is depicted in fig. 2 .

In order to arrive at unambiguous results at exactly $0^{\circ}$, the experimental data were analyzed using a 
Table 2

Absolute $\mathrm{CM}$ cross section values from this experiment, together with their statistical errors in two cases: taking into account the full transmission region, and from the plateau region only. The photon energy bin widths are also indicated.

\begin{tabular}{lrl}
\hline & \multicolumn{1}{l}{$\begin{array}{l}E_{\gamma, \mathrm{Lab}} \\
(\mathrm{MeV})\end{array}$} & $\begin{array}{l}\mathrm{d} \sigma / \mathrm{d} \Omega\left(0^{\circ}\right) \\
\left.(\mu \mathrm{bsr})^{-1}\right)\end{array}$ \\
\hline full transmission region & $\begin{aligned} 8.86 \pm 0.65 \\
10.07 \pm 0.74\end{aligned}$ & $5.49 \pm 0.38$ \\
& $11.51 \pm 0.65$ & $5.74 \pm 0.23$ \\
$13.42 \pm 0.65$ & $6.69 \pm 0.42$ \\
& $15.00 \pm 0.65$ & $5.99 \pm 0.39$ \\
& $16.60 \pm 0.62$ & $6.59 \pm 0.38$ \\
& $18.11 \pm 0.58$ & $7.11 \pm 0.48$ \\
& & \\
plateau region only & $9.98 \pm 1.17$ & $5.38 \pm 0.20$ \\
& $14.71 \pm 1.61$ & $6.11 \pm 0.29$ \\
\hline
\end{tabular}

Monte Carlo simulation of the entire reaction process, taking into account the energy loss and the multiple scattering of the protons in the target, the angular distribution of the emitted particles, and the energy dependence of the cross section. The differential cross sections from two different theoretical calculations were chosen as an input, namely a classical one and a relativistic one [9].

In fig. 2 , the result from such a simulation is shown for $\left\langle E_{\gamma}\right\rangle=10 \mathrm{MeV}$, in which the theoretical zero degree cross section (i.e. the $a+c$ coefficients in the classical Partovi expansion) has been properly adjusted to yield the same spectral area as the experimental one. The standard error on the Monte Carlo calculation is about $0.5 \%$. Moreover, the analysis proved that the resulting experimental cross section

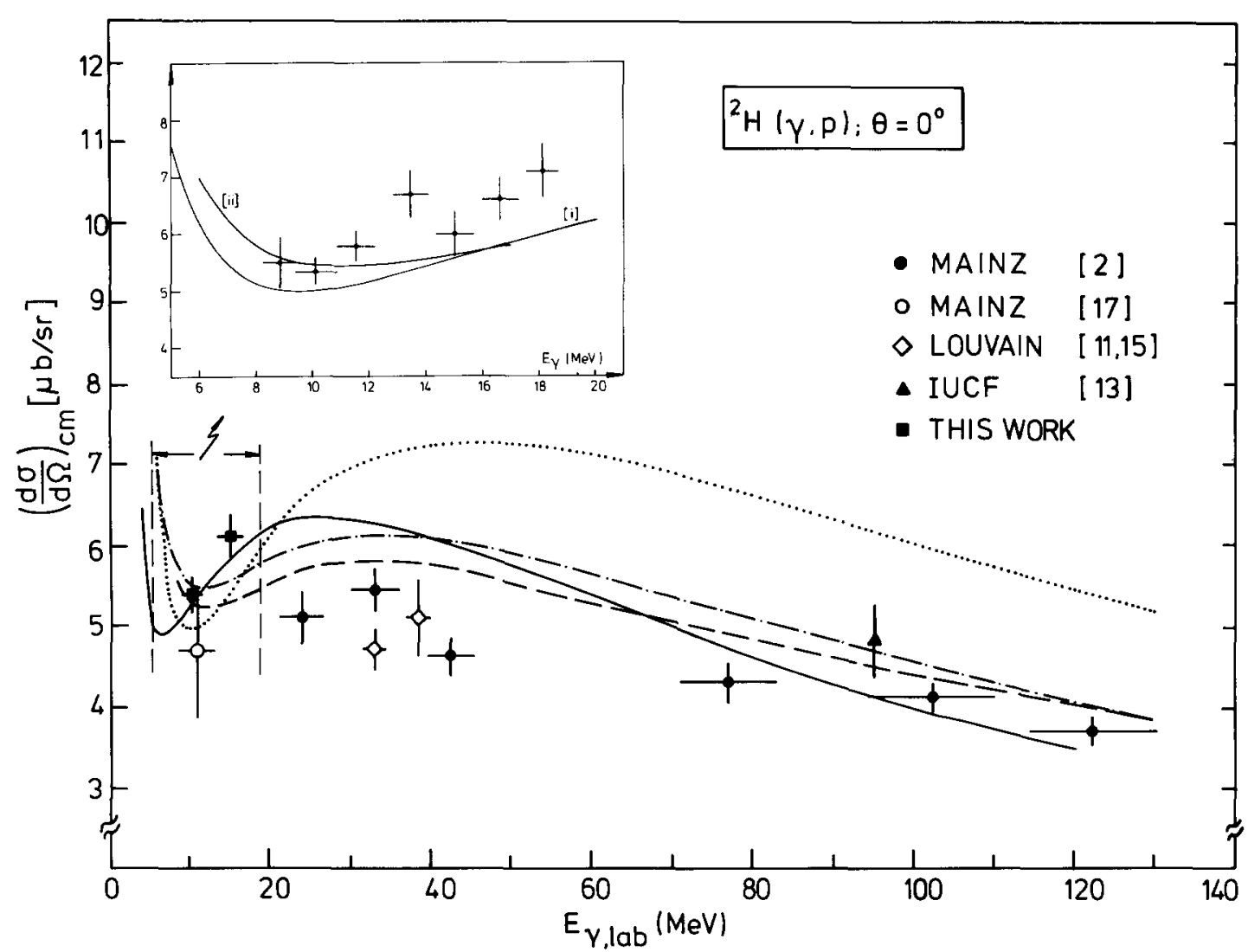

Fig. 3. The forward CM differential cross section for the ${ }^{2} \mathrm{H}(\gamma, \mathrm{p})$ n reaction as a function of lab photon energy, compared with the results from various theoretical approaches; dots: ref. [1], dashes: ref. [7], dash-dots: ref. [6] and full line: ref. [10]. In the insert the absolute cross section values are shown, resulting from the analysis of the entire transmission region (table 2); the curves depict the theoretical calculations [9] (with (i): classical and (ii): relativistic), as used in the simulation (see text). 
values are hardly influenced by the choice of the theoretical input (the deviations between both being smaller than $1 \%$ ).

The deduced absolute $\mathrm{CM}$ cross section values are listed in table 2 . A comparison between experimental and some theoretical calculations $[1,6,7,10]$ is made in fig. 3, showing the existing data points [2,11-17] and our new values from table 2 (plateau region only). Although the value of the forward cross section at $E_{\gamma} \sim 10 \mathrm{MeV}$ is found to be in good agreement with all relativistic approaches, the result at $E_{\gamma} \sim 15$ $\mathrm{MeV}$ is commonly underestimated. The sole calculation that describes both new data points satisfactorily is the one by Nagornyi et al. [10]. In the adjacent energy region - from 20 to $40 \mathrm{MeV}$ - it is furthermore observed that all calculations overshoot the experimental cross section values. In the inset of fig. 3 the low energy region is shown in detail: the set of values corresponding to the full transmission (table 2 ) are plotted together with the theoretical cross sections of ref. [9], used in the analysis procedure. The rising trend of the cross section in the measured energy interval is apparent, suggesting indeed the existence of a minimum.

In order to extract the $\boldsymbol{a}$ and $\boldsymbol{c}$ Partovi coefficients separately and unambiguously, the knowledge of the backward cross section at the same photon energy is demanded. The analysis of such measurements that have been performed with the same experimental setup, is in progress and will be treated in detail in a forthcoming paper. Furthermore, a full discussion will be included of the overall accuracy of these experiments, based upon the measurements of the forward Compton electron yield, again using the same system. Finally, from the trend observed in the present ex- periment and the comparison with theory, it can be concluded that it would be extremely worthwhile to have additional accurate data points both at lower $(<10 \mathrm{MeV})$ and at higher $(>20 \mathrm{MeV})$ photon energies. Such experiments are in progress at the moment.

We acknowledge the financial support lent by the Interuniversity Institute for Nuclear Science (IIKW) and by the National Fund for Scientific Research (NFWO), Brussels, Belgium.

\section{References}

[1] F. Partovi, Ann. Phys. (NY) 27 (1964) 79.

[2] R.J. Hughes, A. Zieger, H. Wäffler and B. Ziegler, Nucl. Phys. A 267 (1976) 329.

[3] E.L. Lomon, Phys. Lett. B 68 (1977) 419

[4] H. Arenhövel, Z. Phys. A 302 (1981) 25.

[5] A. Cambi, B. Mosconi and P. Ricci, Phys. Rev. Lett. 48 (1982) 462; Phys. Rev. C 26 (1984) 2358.

[6] J.L. Friar, B. Gibson and G. Payne, Phys. Rev. C 30 ( 1984 ) 441 ; and private communication.

[7] W. Jaus and S. Woolcock, Nucl. Phys. A 431 (1984) 669; Phys. A 473 (1987) 667, 685.

[8] J.M. Laget, private communication.

[9] P. Wilhelm, W. Leidemann and H. Arenhövel, Few Body Syst. 3 (1988) 111; and private communication.

[10] S.I. Nagornyi, Yu.A. Kasatkin, E.V. Inopin and I. Kirichenko, Yad. Fiz. 44 (1986) 1171; Sov. J. Nucl. Phys. 44 (1986) 760.

[11 ] J.F. Gilot et al., Phys. Rev. Lett. 47 (1981) 304.

[12] A. Ninane et al., Can. J. Phys. 62 (1984) 1104.

[13] H.O. Meyer et al., Phys. Rev. Lett. 52 (1984) 1759.

[14] C. Dupont et al., Nucl. Phys. A 445 (1985) 13.

[15] A. Ninane et al., Phys. Rev. C 35 (1987) 402.

[16] P. Levi-Sandri et al., Phys. Rev. Lett. 59 (1986) 2543.

[17] A. Zieger, P. Grewer and B. Ziegler, Few Body Syst. 1 (1986) 135. 